\title{
The growing pains of
}

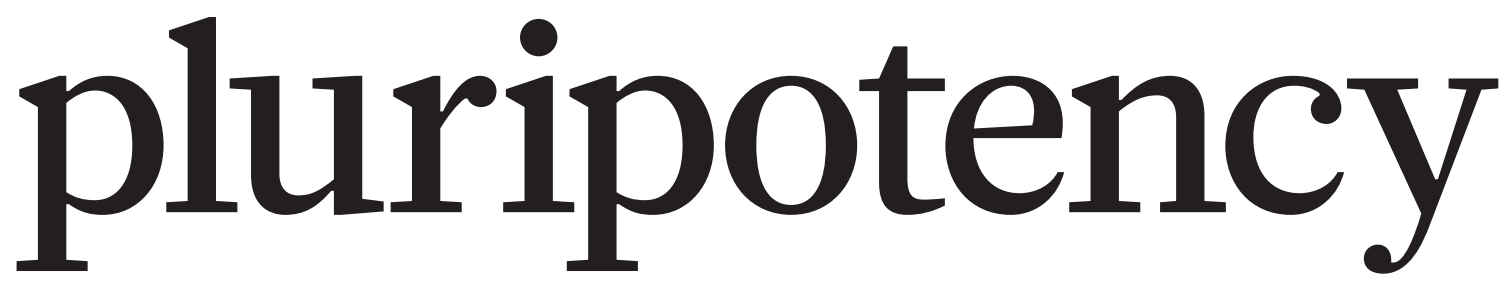

\section{The field of induced pluripotent stem cells has grown up fast. Now it is entering the difficult stage.}

\section{BY ERIKA CHECK HAYDEN}

$\mathbf{F}$ Tive years is an eye-blink in science, but that's all the time it has taken for the concept of adult-cell reprogramming to revolutionize the field of regenerative medicine. In August 2006, Shinya Yamanaka of Kyoto University in Japan told the world that he had turned mouse skin cells into induced pluripotent stem (iPS) cells, capable of becoming many types of cell ${ }^{1}$. The following year, he repeated the feat for human cells ${ }^{2}$.

Like human embryonic stem cells, iPS cells could potentially be used as therapies, disease models or in drug screening. And iPS cells have clear advantages: they can be made from adult cells, avoiding the contentious need for a human embryo, and they can be derived from people with diseases to create models or even therapies based on a person's genetic make-up. Scientists predicted that iPS cells would change the face of biology and medicine - and some would say they already have. In the past year or so, researchers have published cellular models derived from iPS cells for a staggering array of conditions, from heart defects ${ }^{3}$ to schizophrenia ${ }^{4}$. And treatments based on iPS cells are moving toward the clinic: in California, for example, a team hopes to gain approval within the next three years to start treating people with the devastating skin disease epidermolysis bullosa using skin tissue grown from their iPS cells.

Yet work in the past few months has highlighted several potential roadblocks. Reprogramming can be inefficient and induce mutations; the reprogrammed cells cannot develop into some cell types; and those they can generate are not always a good model for disease. New issues are emerging apace: work published last week ${ }^{5}$ shows that, in a particular strain of mice, iPS cells cause immune reactions when they are transplanted into other mice with the same genetic make-up — raising questions about whether it will be possible to transplant iPS-cell-derived tissue back into the person from which it is made. No one doubts that iPS cells still have enormous potential, but the field's initial optimism has cooled. "Right now, we are a long way from being sophisticated enough to take advantage of these cells' potential," says neuroscientist Arnold Kriegstein of the University of California, San Francisco (UCSF). "Things are still at a very early stage." Here, Nature looks at some of the field's biggest challenges, and how they are being tackled.

\section{FINDING A RECIPE}

From the start, biologists have tried to devise safer and more efficient recipes for making iPS cells than Yamanaka's method, which used a retrovirus to deliver a powerful shot of four genetic reprogramming factors into cells. Retroviruses integrate into a host cell's DNA and can therefore potentially disrupt gene expression and lead to cancer; and one of the reprogramming factors, $M y c$, is itself an oncogene that could cause cancer.

To outsiders at least, a new, 'improved' reprogramming method seems to be published every month. But Yamanaka's retroviral method is still the most efficient, and the one used most widely. The retroviral technique can transform about $0.01 \%$ of human skin stem cells into pluripotent cell lines; by comparison, adenoviruses, which do not integrate into the genome, transform just $0.0001-0.0018 \%$ of cells ${ }^{6}$, and delivering the reprogramming factors directly into a human cell transforms $0.001 \%$ (ref. 7). Inefficiency increases the cost and difficulty of deriving iPS cells for cell banks, and poses a particular problem when working with rare cell sources. Researchers have also tried omitting $M y c$, as well as silencing it or stripping it from the cell once reprogramming is complete. But these workarounds also lower the efficiency of reprogramming, and a silenced $M y c$ might be reactivated.

Addressing these concerns is already a top priority for the field. Researchers continue to tinker with their reprogramming recipes, trying to find the factors, and means of delivery, that are the most efficient and don't increase the risk of cancer. In April, a group led by Edward Morrisey at the University of Pennsylvania in Philadelphia reported that it could boost the efficiency of reprogramming by two orders of magnitude over standard techniques by using a retrovirus to shuttle in a cluster of microRNAs. "It is very important for us to get these reprogramming methods to work well enough so that we can compare them and see whether they make any difference in the stability of the cells and in tumorigenicity," $\rightarrow$ NATURE.COM Read more about iPS cells at go.nature.com/otnzrl says developmental neurobiologist Jeanne Loring of The Scripps Research Institute in La Jolla, California. "No one has done that yet, and it is going to be a long haul before we figure this out." 


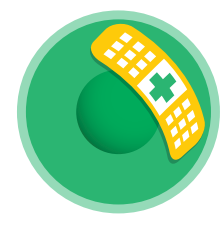

\section{PATCHING THE SCARS}

A whole new set of questions has arisen over the past year, concerning the genetic impact of the reprogramming process. In July 2010, groups led by George Daley ${ }^{9}$ at the Children's Hospital Boston and by Konrad Hochedlinger ${ }^{10}$ at the Massachusetts General Hospital in Cambridge published studies showing that iPS cells carried an 'epigenetic memory' - chemical modifications in their DNA that had come from the original adult cells and had not been erased by the reprogramming. This, they say, explains why iPS cells cannot generate as many adult cell types as embryonic stem cells can.

Researchers were soon reporting that iPS cells were more likely to contain mutations than cultured human embryonic stem cells. Four groups scoured the genomes of iPS cells for changes in single DNA bases ${ }^{11}$, DNA rearrangements called copy-number variations ${ }^{12,13}$ and differences in chromosome number ${ }^{14}$. The studies found higher levels of all three. Worse, the mutations in iPS cells were not just inherited from the parent cells - some seemed to result from the reprogramming and culture process. Loring's group reported ${ }^{12}$, for instance, that a protocol for differentiating iPS cells into cardiac cells selected for cells with genetic rearrangements.

This picture is still coming together. One of the studies ${ }^{13}$ on copy-number variations found that many of the rearrangements disappeared after the iPS cells were cultured over long periods of time, probably because the most severely mutated cells were outcompeted by the genetically healthier ones. But this February, a team led by Joseph Ecker at the Salk Institute for Biological Studies in La Jolla, California, reported that it had detected epigenetic signatures of the parent cells in human iPS cell lines even after they had been cultured many times and differentiated into specific cell types ${ }^{15}$. A third study ${ }^{16}$ suggested that iPS cells are no worse than embryonic stem cells in this regard. Developmental biologist Alexander Meissner of Harvard University in Cambridge and his team reported that epigenetic and genetic variation was similar across 20 human embryonic stem cell lines and 12 iPS cell lines. "What we see is not so much a lot of variation across iPS cells, but a lot of variation across pluripotent cells," Meissner says.

Researchers expressed their concerns about such effects at a meeting convened by the US National Institutes of Health and the Food and Drug Administration (FDA) in Bethesda, Maryland, on 21-22 March that focused on hurdles to translating research on pluripotent cells into the clinic. The concern is that the mutations could have unpredictable and undesirable effects on the cells, and on the patients they end up in. "The genomic changes are going to be a big deal to the FDA," Loring says.

Meissner's group has devised a 'scorecard' of gene expression and methylation - a type of epigenetic mark - that correlates with an iPS cell line's level of pluripotency. It should help researchers to identify and avoid the practices that generate the worst genetic aberrations, and to screen for the lines that are least affected. And researchers are beginning to examine whether and how these genetic and epigenetic effects affect the capabilities and characteristics of iPS cells.

"Right now there are two schools of thought on this," Loring says. "One is that the sky is falling, and the other is that it's a good thing that we're finding out about this now, so that we can discover whether these are biologically relevant changes."

\section{HITTING THE LIMITS}

iPS cells are immensely flexible, but they can't do everything. Liver cells derived from iPS cells could in theory replace animals in drug toxicology screening, for example. But researchers have struggled to get any human stem cells to differentiate into tissues, such as liver, that are normally derived from the endoderm - the most interior of the three germ layers that make up the embryo. This might be because the series of signals necessary for these cells' development and complex function are difficult to recapitulate. Liver specialist Holger Willenbring at the UCSF points out that hepatocytes have many roles, from detoxifying the blood to making circulating proteins. "There are a lot of different jobs that the cell has to accomplish, and it is hard to get that right in cell culture," he says.

One of the hottest areas of research on human stem cells is in cell-replacement therapy for type-1 diabetes, a disease that develops when insulin-producing cells in the pancreas are destroyed. But no one has been able to make a fully functional and mature insulin-producing pancreatic beta cell - also derived from the endoderm - because researchers don't know the exact series of growth signals necessary and, perhaps, because beta cells usually develop in a three-dimensional environment that is difficult to replicate in a culture dish. Maybe this won't matter, says developmental biologist Matthias Hebrok at the UCSF. Beta-cell 'progenitors' have been made, from embryonic stem cells and from iPS cells, that can secrete insulin. They are less efficient than a normal beta cell, but perhaps efficient enough to help a person with diabetes.

Researchers are making a concerted effort to devise the correct recipe for making mature beta cells and hepatocytes, and Willenbring and others say that those in the field are working as a team on this for the first time. A paper published last week ${ }^{17}$ circumvented iPS cells altogether, describing the generation of hepatocyte-like cells directly from mouse skin cells, using a cocktail of regulatory proteins important for liver development. But Willenbring says he still has questions about whether the cells were able to perform all the functions of hepatocyes.

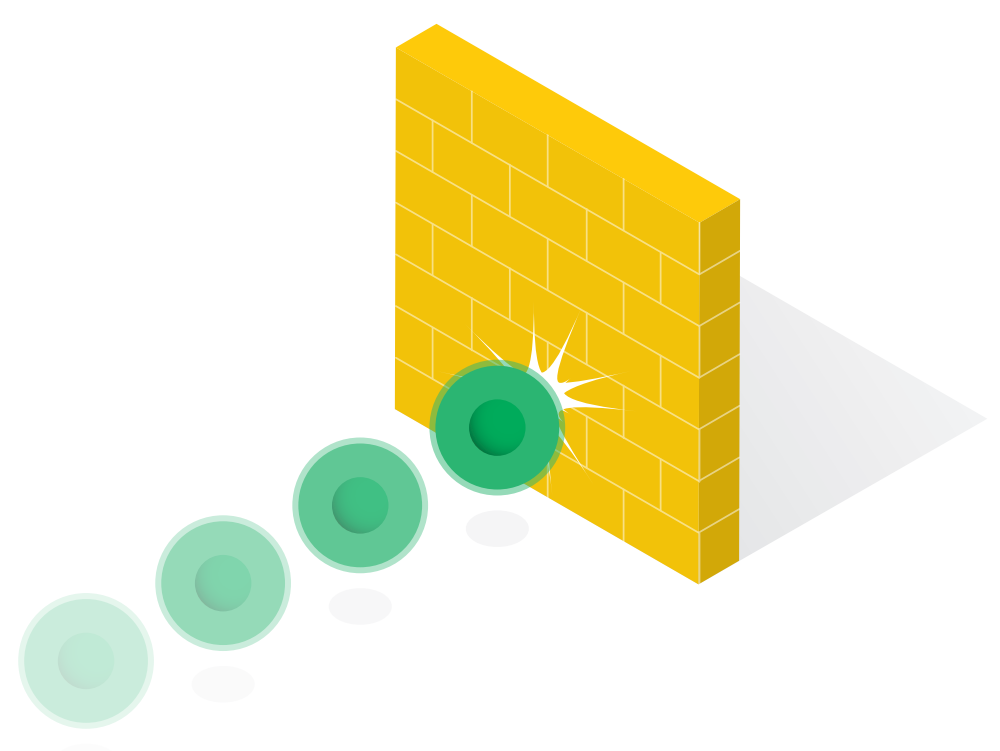




\section{MAINTAINING STANDARDS}

The relative ease of reprogramming has thrown the iPS cell field open to almost anyone. But from the start, researchers have worried that the low barrier to entry and the extremely competitive pace have meant that standards are not as rigorous as they should be. "There's a tremendous amount of pressure to get these papers out there, and in the rush investigators are not characterizing their cells very well," Kriegstein says.

Kriegstein points to a paper published last November ${ }^{18}$ by a team led by Alysson Muotri of the University of California, San Diego. The team studied people with a mutated gene that causes the neurological condition Rett syndrome as a model for an autism-spectrum disorder, which causes behavioural difficulties. The researchers derived iPS cells from these patients, and showed that as the iPS cells differentiated into neurons, they initially expressed genes typically found in neural 'precursor' cells, then later expressed genes involved in neuronal signalling. The neurons from the patients with Rett syndrome were smaller than those derived from people without the disease, and they also had signalling defects and other differences. But Kriegstein says that this molecular characterization is not enough to show exactly what type of neurons had formed, what part of the central nervous system they represented and how they therefore relate to processes that go awry in the brain. "Possibly the reported abnormalities are relevant to the intended diseases," Kriegstein says, referring to this and other iPS papers, but this would be very surprising given that most neurodevelopmental and neurodegenerative diseases affect specific populations of neurons at specific times during development, he says.

Muotri says that his team's analysis of the neurons was thorough, and included electrophysiological tests showing that the cells could fire action potentials and were therefore functional. $\mathrm{He}$ also points out that almost all neurons in the brain express the gene, so he did not want to limit the studies just to regions that had been associated with Rett syndrome, as insights from the cultured neurons could illuminate the molecular mechanisms that disrupt the circuits controlling behaviour. "When we find such a cellular phenotype in culture, we know we can now start from there to understand other layers of complexity," Muotri says.

\section{MODELLING THE MIND IS HARD}

Investigators are creating patient-specific iPS cells to model almost every disease known - but in some cases, researchers question how much can be learned from the models. The biggest debate is over models of complex neuropsychiatric and behavioural disorders: can reprogrammed cells really mimic conditions such as schizophrenia or autism, which affect the brain and behaviour in complicated ways? "I've had clinicians ask if we can make iPS cells from a patient who was mentally retarded," says developmental biologist Christine Mummery of the Leiden University Medical Center in the Netherlands. But she questions how useful that would be. "I said, 'I don't know, how you would measure the IQ of a neuron in a dish?'”

Researchers working with these models argue that they are still valuable. In April, Fred Gage at the Salk Institute and his team reported ${ }^{4}$ that they had derived neurons from the skin cells of a person with schizophrenia, and that some differences between those neurons and normal neurons could be corrected by administration of the antipsychotic drug loxapine. Like Muotri, Gage says that the model is designed to uncover how the genetic factors underlying schizophrenia affect the function of neurons. "Although we cannot measure the behaviour of the patients, we propose that we can measure the activity of the neurons, and the goal is to search for cellular and molecular processes that underlie

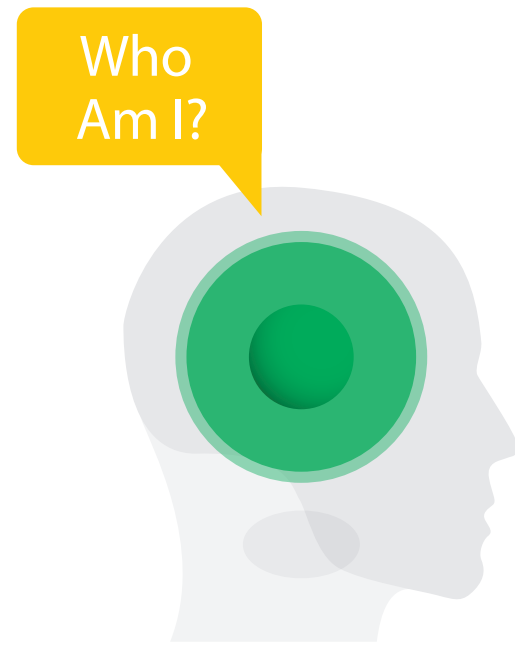

the behavioural phenotypes," he says.

A related problem arises with diseases of ageing, a hot field in iPS-cell research: many of the conditions strike mature cells, so iPS cells - which are essentially starting their developmental lives afresh - might not be relevant. With a disease such as Parkinson's, says Mummery, "this is a real issue - will you be able to get neurons mature enough to see anything? People are working very hard not only to make their cell type of interest but also to make them mature, so that's still a major technical obstacle."

Some researchers counter that even 'young' cells show traits related to diseases of ageing. Renee Reijo Pera at Stanford University in California made iPS cells from Genia Brin - the mother of Google co-founder Sergey Brin. She has Parkinson's, a condition that is marked by the destruction of dopamine-producing neurons. Once differentiated into neurons, the cells secreted dopamine and were more sensitive to chemicals that can induce cell death than were dopamine-secreting neurons derived from healthy people ${ }^{19}$. "This seems to be to be the best model of Parkinson's disease," Reijo Pera says.

Many iPS-cell researchers see the field's growing pains as signs that it is reaching a state of maturity; they say that the problems are no different from those that many biomedical research fields face as they inch towards clinical application. "There was this huge euphoria in the beginning, with everyone thinking iPS will do everything, cure all diseases, and be super-easy," Meissner says.

"But not everyone can become a stem-cell biologist overnight," he says. "It's a bit of a reality check that things are not as simple as we thought."

Erika Check Hayden is a senior reporter for Nature based in San Francisco.

1. Takahashi, K. \& Yamanaka, S. Cell 126, 663-676 (2006).

2. Takahashi, K. et al. Cell 131, 861-872 (2007).

3. Itzhaki, I. et al. Nature 471, 225-229 (2011).

4. Brennand, K. J. et al. Nature 473, 221-225 (2011).

5. Zhao, T., Zhang, Z.-N., Rong, Z. \& Xu, Y. Nature advance online publication doi:10.1038/nature10135 (2011).

6. González, F., Boué, S. \& Izpisúa Belmonte, J. C. Nature Rev. Genet. 12, 231-242 (2011).

7. Kim, D. et al. Cell Stem Cell 4, 472-476 (2009)

8. Anokye-Danso, F. et al. Cell Stem Cell 8, 376-388 (2011).

9. Kim, K. et al. Nature 467, 285-290 (2010).

10. Polo, J. M. et al. Nature Biotechnol. 28, 848-855 (2010).

11.Gore, A. et al. Nature 471, 63-67 (2011).

12.Laurent, L. C. et al. Cell Stem Cell 8, 106-118 (2011).

13. Hussein, S. M. et al. Nature 471, 58-62 (2011).

14. Mayshar, Y. et al. Cell Stem Cell 7, 521-531 (2010)

15. Lister, R. et al. Nature 471, 68-73 (2011).

16. Bock, C. et al. Cell 144, 439-452 (2011).

17.Huang, P. et al. Nature advance online publication doi:10.1038/ nature10116 (2011).

18. Marchetto, M. C. N. et al. Cell 143, 527-539 (2010).

19.Nguyen, H. N. et al. Cell Stem Cell 8, 267-280 (2011). 